\title{
Genome-wide association study for refractive astigmatism reveals genetic co-determination with spherical equivalent refractive error: the CREAM consortium
}

\author{
Qing Li · Robert Wojciechowski · Claire L. Simpson · Pirro G. Hysi • Virginie J. M. Verhoeven • \\ Mohammad Kamran Ikram • René Höhn • Veronique Vitart • Alex W. Hewitt • Konrad Oexle • Kari-Matti Mäkelä • \\ Stuart MacGregor · Mario Pirastu • Qiao Fan · Ching-Yu Cheng • Beaté St Pourcain · George McMahon · \\ John P. Kemp · Kate Northstone · Jugnoo S. Rahi · Phillippa M. Cumberland • Nicholas G. Martin • \\ Paul G. Sanfilippo · Yi Lu · Ya Xing Wang • Caroline Hayward · Ozren Polašek · Harry Campbell · Goran Bencic • \\ Alan F. Wright · Juho Wedenoja · Tanja Zeller · Arne Schillert • Alireza Mirshahi · Karl Lackner · Shea Ping Yip • \\ Maurice K. H. Yap · Janina S. Ried · Christian Gieger · Federico Murgia · James F. Wilson • Brian Fleck · \\ Seyhan Yazar · Johannes R. Vingerling • Albert Hofman • André Uitterlinden · Fernando Rivadeneira • \\ Najaf Amin • Lennart Karssen - Ben A. Oostra $\cdot$ Xin Zhou $\cdot$ Yik-Ying Teo $\cdot$ E. Shyong Tai $\cdot$ Eranga Vithana $\cdot$ \\ Veluchamy Barathi · Yingfeng Zheng • Rosalynn Grace Siantar · Kumari Neelam • Youchan Shin · Janice Lam • \\ Ekaterina Yonova-Doing • Cristina Venturini · S. Mohsen Hosseini · Hoi-Suen Wong · Terho Lehtimäki • \\ Mika Kähönen · Olli Raitakari · Nicholas J. Timpson · David M. Evans · Chiea-Chuen Khor · Tin Aung • \\ Terri L. Young · Paul Mitchell • Barbara Klein · Cornelia M. van Duijn · Thomas Meitinger · Jost B. Jonas • \\ Paul N. Baird · David A. Mackey · Tien Yin Wong • Seang-Mei Saw · Olavi Pärssinen · Dwight Stambolian • \\ Christopher J. Hammond · Caroline C. W. Klaver · Cathy Williams · Andrew D. Paterson · Joan E. Bailey-Wilson • \\ Jeremy A. Guggenheim • The CREAM Consortium
}

Received: 14 April 2014 / Accepted: 30 September 2014 / Published online: 4 November 2014 (C) The Author(s) 2014. This article is published with open access at Springerlink.com

\begin{abstract}
To identify genetic variants associated with refractive astigmatism in the general population, metaanalyses of genome-wide association studies were performed for: White Europeans aged at least 25 years (20 cohorts, $N=31,968$ ); Asian subjects aged at least 25 years
\end{abstract}

S.-M. Saw, O. Pärssinen, D. Stambolian, C. J. Hammond, C. C. W. Klaver, C. Williams, A. D. Paterson, J. E. BaileyWilson, and J. A. Guggenheim jointly directed this work.

The members of the CREAM Consortium are listed in "Appendix"

Electronic supplementary material The online version of this article (doi:10.1007/s00439-014-1500-y) contains supplementary material, which is available to authorized users.

Q. Li · R. Wojciechowski · C. L. Simpson · J. E. Bailey-Wilson National Human Genome Research Institute, National Institutes of Health, 333 Cassell Drive Suite 1200, Baltimore,

MD 21224, USA

R. Wojciechowski

Department of Epidemiology, Johns Hopkins Bloomberg School of Public Health, Baltimore, MD, USA
(7 cohorts, $N=9,295)$; White Europeans aged $<25$ years ( 4 cohorts, $N=5,640$ ); and all independent individuals from the above three samples combined with a sample of Chinese subjects aged $<25$ years $(N=45,931)$. Participants were classified as cases with refractive astigmatism if the average cylinder power in their two eyes was at least 1.00 diopter and as controls otherwise. Genome-wide association analysis was carried out for each cohort separately using logistic regression. Meta-analysis was conducted using a fixed effects model. In the older European group the most strongly associated marker was downstream of the neurexin-1 (NRXN1) gene (rs1401327, $P=3.92 \mathrm{E}-8)$. No other region reached genome-wide significance, and association signals were lower for the younger European group and Asian

\footnotetext{
R. Wojciechowski

Wilmer Eye Institute, Johns Hopkins Medical Institutions,

Baltimore, MD, USA

P. G. Hysi - E. Yonova-Doing - C. Venturini - C. J. Hammond Department of Twin Research and Genetic Epidemiology, King's College London, St Thomas' Hospital Campus, London, UK
} 
group. In the meta-analysis of all cohorts, no marker reached genome-wide significance: The most strongly associated regions were, NRXN1 (rs1401327, $P=2.93 \mathrm{E}-07$ ), TOX (rs7823467, $P=3.47 \mathrm{E}-07$ ) and LINC00340 (rs12212674, $P=1.49 \mathrm{E}-06$ ). For 34 markers identified in prior GWAS for spherical equivalent refractive error, the beta coefficients for genotype versus spherical equivalent, and genotype versus refractive astigmatism, were highly correlated $(r=-0.59, P=2.10 \mathrm{E}-04)$. This work revealed no consistent or strong genetic signals for refractive astigmatism; however, the TOX gene region previously identified in GWAS for spherical equivalent refractive error was the second most strongly associated region. Analysis of additional markers provided evidence supporting widespread genetic co-susceptibility for spherical and astigmatic refractive errors.

\section{Introduction}

Refractive astigmatism results from the optical summation of the eye's corneal astigmatism and astigmatism from internal eye components (e.g. lens). In most individuals, these two sources of astigmatism tend to compensate for each other, such that overall refractive astigmatism is typically low in magnitude (Kelly et al. 2004). High levels of refractive astigmatism are usually the result of high corneal astigmatism rather than high internal astigmatism (Keller et al. 1996; Kee 2013). Astigmatism in infancy is a risk factor for amblyopia (Abrahamsson and Sjostrand 2003). In later life,

V. J. M. Verhoeven · J. R. Vingerling · C. C. W. Klaver Department of Ophthalmology, Erasmus Medical Center, Rotterdam, The Netherlands

V. J. M. Verhoeven · A. Hofman · A. Uitterlinden ·

F. Rivadeneira - N. Amin · L. Karssen · C. M. van Duijn ·

C. C. W. Klaver

Department of Epidemiology, Erasmus Medical Center,

Rotterdam, The Netherlands

M. K. Ikram · C.-Y. Cheng · E. Vithana · V. Barathi · Y. Zheng ·

R. G. Siantar · K. Neelam · Y. Shin · J. Lam · T. Aung ·

T. Y. Wong · S.-M. Saw

Singapore Eye Research Institute, Singapore, Singapore

M. K. Ikram · C.-Y. Cheng · E. Vithana · V. Barathi · C.-C. Khor ·

T. Y. Wong $\cdot$ S.-M. Saw

Department of Ophthalmology, Yong Loo Lin School

of Medicine, National University of Singapore, Singapore, Singapore

M. K. Ikram · C.-Y. Cheng · T. Y. Wong

Office of Clinical Sciences, Duke-NUS Graduate Medical

School, Singapore, Singapore

R. Höhn · A. Mirshahi

Department of Ophthalmology, University Medical Center

Mainz, Mainz, Germany astigmatism commonly accompanies myopia and hyperopia (Mandel et al. 2010; Kee et al. 2005; Farbrother et al. 2004), reducing visual acuity unless corrected by spectacles, contact lenses or refractive surgery (Read et al. 2007).

The results of twin (Dirani et al. 2008; Grjibovski et al. 2006; Parssinen et al. 2012; Teikari and O'Donnell 1989), family (Rakhshani et al. 2012; Mash et al. 1975) and molecular genetic studies (Fan et al. 2011; Lopes et al. 2013; Mackey et al. 2011) suggest that astigmatism is highly heritable, as does its high prevalence in specific ethnic groups such as Native Americans (McKean-Cowdin et al. 2011; Mohindra and Nagaraj 1977; Harvey et al. 2010). For refractive astigmatism, the heritability has been estimated at 0.33 to 0.63 from twin studies (Hammond et al. 2001; Grjibovski et al. 2006; Parssinen et al. 2013). Using a case-control genome-wide association study (GWAS) meta-analysis of 8,513 individuals of Asian ethnicity, Fan et al. (2011) identified the PDGFRA gene on chromosome $4 \mathrm{q} 12$ as a susceptibility locus for corneal astigmatism. Cases were defined as subjects with corneal astigmatism (averaged between the two eyes) of at least 0.75 diopters (D) and controls as those with corneal astigmatism less than $0.75 \mathrm{D}$. Three single nucleotide polymorphisms (SNPs) attained genome-wide significance $(P<5.0 \mathrm{E}-08)$; rs7677751, rs2307049 and rs7660560. SNPs in the same region of PDGFRA have since been found to be associated with both corneal curvature and axial length (Han et al. 2011; Guggenheim et al. 2013; Mishra et al. 2012), but not with spherical refractive error (Guggenheim et al. 2013).

R. Höhn

Klinik Pallas, Olten, Switzerland

V. Vitart · C. Hayward · A. F. Wright

Medical Research Council Human Genetics Unit, Institute of Genetics and Molecular Medicine, University of Edinburgh, Edinburgh, EH4 2XU, UK

A. W. Hewitt · P. N. Baird · D. A. Mackey

Centre for Eye Research Australia, University of Melbourne,

Royal Victorian Eye and Ear Hospital, Melbourne, Australia

A. W. Hewitt · P. G. Sanfilippo · S. Yazar · D. A. Mackey Centre for Ophthalmology and Visual Science, Lions Eye Institute, University of Western Australia, Perth, Australia

K. Oexle $\cdot$ T. Meitinger

Institute of Human Genetics, Klinikum rechts der Isar,

Technische Universität München, Munich, Germany

K.-M. Mäkelä · T. Lehtimäki

Department of Clinical Chemistry, Filmlab laboratories,

Tampere University Hospital and School of Medicine, University of Tampere, 33520 Tampere, Finland

S. MacGregor · Y. Lu

Statistical Genetics, QIMR Berghofer Medical Research Institute Royal Brisbane Hospital, Brisbane, Australia 
A second GWAS meta-analysis in 22,100 individuals of European descent by Lopes et al. (2013) reported suggestive evidence that SNPs in the VAX2 gene on chromosome 2p13 also confer susceptibility to refractive astigmatism (most strongly associated SNP, rs3771395; $P=2.0 \mathrm{E}-07$ ). These authors modelled astigmatism as a continuous trait, using an inverse normal transformation of the refractive astigmatism averaged between the two eyes.

The GWAS meta-analyses of Fan et al. (2011) and Lopes et al. (2013) both assessed large numbers of individuals derived from cohorts that were largely population based. It is therefore unlikely that common autosomal genetic variants, i.e. those with a minor allele frequency (MAF) $>5 \%$, with profound effects on the risk of developing astigmatism (e.g. OR > 2) exist, as both studies would have had high power to detect them. Instead, the results of the two studies imply that most of the additive genetic risk for astigmatism arises from the combined action of a large number of individual risk variants, each with a small effect. This scenario, which also holds for spherical refractive error (Solouki et al. 2010; Hysi et al. 2010; Verhoeven et al. 2013b; Kiefer et al. 2013), suggests that substantially increasing the sample size of GWAS meta-analyses will be an effective method of discovering new variants, albeit with increasingly diminishing returns (Lango Allen et al. 2010). Here, we describe the largest GWAS for refractive astigmatism yet undertaken involving almost 46,000 persons.

M. Pirastu · F. Murgia

Institute of Population Genetics CNR, Traversa La Crucca,

3-07040 Reg. Baldinca, Li Punti, Sassari, Italy

Q. Fan $\cdot$ X. Zhou $\cdot$ Y.-Y. Teo $\cdot$ E. S. Tai $\cdot$ S.-M. Saw

Saw Swee Hock School of Public Health, National University

of Singapore, Singapore, Singapore

B. St Pourcain · G. McMahon - J. P. Kemp · N. J. Timpson •

D. M. Evans

MRC Integrative Epidemiology Unit (IEU), University of Bristol, Bristol BS8 2BN, UK

B. St Pourcain - G. McMahon - J. P. Kemp · K. Northstone ·

N. J. Timpson · D. M. Evans · C. Williams

School of Social and Community Medicine, University of Bristol, Bristol BS8 2BN, UK

J. S. Rahi · P. M. Cumberland

Centre of Epidemiology and Biostatistics, UCL Institute of Child

Health, London, UK

J. S. Rahi $\cdot$ C. Venturini

Institute of Ophthalmology, University College London, London, UK

J. S. Rahi · P. M. Cumberland

Ulverscroft Vision Research Group, UCL Institute of Child

Health, London, UK

\section{Methods}

Selection of studies for inclusion in the meta-analysis

The CREAM consortium comprises researchers from more than 30 research groups who share a common interest in the genetics of refractive error. From March to July 2012, all Principal Investigators (PIs) of studies known to CREAM members who had collected refractive error phenotype information and genome-wide genotyping information on a study sample were invited to join CREAM. An analysis plan detailing the protocol for the astigmatism GWAS meta-analysis was circulated, inviting all PIs to perform the requested analyses and to submit GWAS results for their study sample. There were no restrictions on which studies were eligible to join the meta-analysis.

Study cohorts and meta-analysis overview

GWAS results were meta-analysed for a total of 32 cohorts. The subject demographics of the cohorts are summarised in Table 1: Further details can be found in the Supplement and the previous publications (Rahi et al. 2011; Fraser et al. 2012; Boyd et al. 2013; Vitart et al. 2010; Parssinen et al. 2010; Sperduto et al. 1996; Foong et al. 2007; Foran et al. 2003; Cornes et al. 2012; Hofman et al. 2011; Burdon et al. 2011; Khor et al. 2011; Oexle et al. 2011; Vithana et al. 2011; Paterson et al. 2010; Klein et al. 2010; Mackey

N. G. Martin

Genetic Epidemiology, QIMR Berghofer Medical Research Institute Royal Brisbane Hospital, Brisbane, Australia

Y. X. Wang

Beijing Institute of Ophthalmology, Beijing Tongren Hospital,

Capital University of Medical Science, Beijing, China

O. Polašek

Faculty of Medicine, University of Split, Split, Croatia

H. Campbell · J. F. Wilson

Centre for Population Health Sciences, University of Edinburgh,

Edinburgh EH8 9AG, UK

G. Bencic

Department of Ophthalmology, Sisters of Mercy University Hospital, Zagreb, Croatia

J. Wedenoja

Department of Public Health, Hjelt Institute, University

of Helsinki, Helsinki, Finland

J. Wedenoja

Department of Ophthalmology, Helsinki University Central

Hospital, Helsinki, Finland 
et al. 2009; Nelis et al. 2009; Raitakari et al. 2008; Spector and Williams 2006; Wichmann et al. 2005; Pardo et al. 2005; Aulchenko et al. 2004; Clemons et al. 2003; Kassoff et al. 1999, 2001; Mitchell et al. 1995; Shamoon et al. 1993). The mean age of the participants in each cohort varied from 15 to 74 years and 37,608 of them were of White European ancestry while 10,212 were of Asian ancestry. Because the magnitude and axis of astigmatism are known to vary with age (Anstice 1971; Lyle 1971), and to limit the effects of differing SNP-causal variant relationships across ethnicities, meta-analyses were carried out separately for (a) White Europeans aged <25 years, (b) White Europeans aged $\geq 25$ years, and (c) Asians aged $\geq 25$ years. This age classification scheme follows that adopted previously by the CREAM consortium (Verhoeven et al. 2013a, b), and was agreed to by the CREAM Executive Committee prior to commencement of the meta-analyses. A final metaanalysis was performed combining all independent samples from these three groups with the SCORM study of Asians aged $<25$ years. Each participating study defined the astigmatism trait in the same manner and performed association analyses specifically for this study using equivalent logistic regression models (described below and in the supplement).

\section{Phenotypic assessment}

Subjects underwent an ophthalmic examination that included either subjective refraction, cycloplegic autorefraction or

\section{T. Zeller}

University Heart Center Hamburg, Clinic for general

and interventional Cardiology, Hamburg, Germany

\section{A. Schillert}

Institute for Medical Biometry and Statistics, Universität zu

Lübeck, University Hospital Schleswig-Holstein, Campus

Lübeck, Lübeck, Germany

\section{A. Schillert}

DZHK (German Centre for Cardiovascular Research), partner site Hamburg/Kiel/Lübeck, Lübeck, Germany

\section{A. Mirshahi}

Dardenne Eye Hospital, Bonn, Germany

\section{K. Lackner}

Institute of Clinical Chemistry and Laboratory Medicine,

University Medical Center Mainz, Mainz, Germany

\section{S. P. Yip}

Department of Health Technology and Informatics, Hong Kong Polytechnic University, Hong Kong SAR, China

S. P. Yip · M. K. H. Yap · J. A. Guggenheim $(\square)$ Centre for Myopia Research, School of Optometry, Hong Kong Polytechnic University, Hung Hom, Kowloon, Hong Kong SAR, China

e-mail: jeremy.guggenheim@ @olyu.edu.hk non-cycloplegic autorefraction (Supplemental Methods and Supplemental Table S1a). Astigmatism was defined in the same way during association analysis in all cohorts participating in this meta-analysis study. Participants with conditions that could alter refraction, such as cataract surgery, laser refractive procedures, retinal detachment surgery, keratoconus or ocular or systemic syndromes were excluded. Additional exclusion criteria were, firstly, a cylinder power $\geq 5.00 \mathrm{D}$ in either eye (to exclude subjects with undiagnosed keratoconus or potential measurement errors), and secondly, a difference in cylinder power between the two eyes beyond four standard deviations from the mean (except for subjects with data for only one eye). Subjects were classified as astigmatic cases if the average cylinder power in the two eyes was $\geq 1.00 \mathrm{D}$ and as controls otherwise (note that cylinder axis was ignored). The threshold value of $1.00 \mathrm{D}$ was chosen due to its common usage in prior work (Read et al. 2007; Huynh et al. 2007). The average of the two eyes was taken to maximise statistical power (Carbonaro et al. 2009).

\section{Genotyping and genotype imputation}

Genotyping and imputation were carried out as described previously (Verhoeven et al. 2013b). In brief, participants in each cohort were genotyped using a whole genome SNP platform. The genotypes of subjects that passed a series of quality control (QC) filters, including call rate at least $>95 \%$ and ancestry matching that of the reference

\section{J. S. Ried · C. Gieger \\ Institute of Genetic Epidemiology, Helmholtz Zentrum München, Neuherberg, Germany \\ B. Fleck \\ Princess Alexandra Eye Pavilion, Edinburgh EH3 9HA, UK}

A. Hofman · A. Uitterlinden · F. Rivadeneira

Netherlands Consortium for Healthy Ageing, Netherlands

Genomics Initiative, The Hague, The Netherlands

A. Uitterlinden $\cdot$ F. Rivadeneira

Department of Internal Medicine, Erasmus Medical Center, Rotterdam, The Netherlands

B. A. Oostra

Department of Clinical Genetics, Erasmus Medical Center, Rotterdam, The Netherlands

Y.-Y. Teo

Department of Statistics and Applied Probability, National

University of Singapore, Singapore, Singapore

E. S. Tai

Department of Medicine, National University of Singapore and National University Health System, Singapore, Singapore 
population, were imputed to a common set of markers (HapMap Phase 2) with either MACH (Li et al. 2010) or IMPUTE (Howie et al. 2012). SNPs that passed cohortspecific QC metrics were used as a framework for imputation, and reference haplotypes were chosen from the best available HapMap Phase 2 ancestry group (Verhoeven et al. 2013b). See Supplemental Methods and Table S1b for more details.

\section{Statistical analysis}

A GWAS was carried out separately for each participant cohort. SNPs were tested individually for association with astigmatism in a logistic regression model, with case/control status as the dependent variable. SNP imputed dosage was modelled as a linear covariate (on a continuous scale from 0 to 2) where one allele was assigned as the reference allele and the other allele the risk allele. Age and sex were included as additional covariates when appropriate. If significant population stratification was detected in a cohort, then either the first two principal components (PCs) were included in the logistic regression or an analysis method was used that jointly adjusted for population stratification and cryptic relatedness as part of the analysis. This approach is commonly used in GWAS meta-analysis (Eeles et al. 2009; Chen et al. 2012; Wang et al. 2012). Details of the GWAS analyses performed in each cohort are given in Supplemental Methods. SNPs were carried forward for

E. S. Tai $\cdot$ V. Barathi $\cdot$ T. L. Young $\cdot$ S.-M. Saw

Duke-National University of Singapore Graduate Medical

School, Singapore, Singapore

E. Vithana

Neuroscience and Behavioural Disorders (NBD) Program, Duke-

NUS Graduate Medical School, Singapore, Singapore

S. M. Hosseini $\cdot$ H.-S. Wong · A. D. Paterson

Genetics and Genome Biology Program, The Hospital for Sick

Children Research Institute, PGCRL Rm 12.9835, 686 Bay

Street, Toronto, ON M5G 0A4, Canada

M. Kähönen

Department of Clinical Physiology, Tampere University Hospital and School of Medicine, University of Tampere, 33521 Tampere, Finland

O. Raitakari

Research Centre of Applied and Preventive Cardiovascular

Medicine, University of Turku, Turku, Finland

O. Raitakari

Department of Clinical Physiology and Nuclear Medicine, Turku

University Hospital, 20041 Turku, Finland

D. M. Evans

Translational Research Institute, University of Queensland

Diamantina Institute, Brisbane, QLD, Australia meta-analysis if they met the following criteria of a MAF $>1 \%$, and an OR (odds ratio) between 0.2 and 5.0 (the latter step being included to remove SNPs with an OR of approximately zero or infinity, which occurred for a few SNPs in the smaller cohorts due to low minor allele counts). Effect estimates were reported with reference to the positive strand of the NCBI Build 36 reference sequence of the human genome. Meta-analysis was carried out using a fixed effects model with METAL (Willer et al. 2010). For the meta-analysis of all cohorts, the adult ALSPAC sample was excluded because, given the inclusion of the ALSPAC young persons sample (biological relatives of the adults), this could have led to falsely inflated estimates of association. The number of subjects contributing information to the meta-analysis summary statistic varied, as shown in Tables 2 and 3. This occurred primarily through markers being monomorphic (uninformative) in certain samples, and to a small extent through missing data for certain markers in specific individuals. A $P$ value $<5.0 \mathrm{E}-08$ was used to declare genome-wide significance (Dudbridge and Gusnanto 2008; Evangelou and Ioannidis 2013).

\section{Results}

Meta-analyses of refractive astigmatism GWAS results were carried out for three subject groups: White Europeans aged $\geq 25$ years, White European subjects aged $<25$ years,

\section{C.-C. Khor}

Division of Human Genetics, Genome Institute of Singapore,

Singapore, Singapore

T. L. Young

Duke Eye Center, Duke University School of Medicine, Durham, NC, USA

P. Mitchell

University of Sydney, Sydney, Australia

P. Mitchell

Western Sydney Local Health Network, Sydney, Australia

P. Mitchell

Westmead Millennium Institute, Westmead, Australia

B. Klein

Ophthalmology and Visual Sciences, Ocular Epidemiology,

University of Wisconsin-Madison, 610 North Walnut Street,

Room 409, Madison, WI 53726, USA

\section{J. B. Jonas}

Beijing Institute of Ophthalmology, Beijing Tongren Eye Center, Beijing Tongren Hospital, Capital Medical University, Beijing Ophthalmology and Visual Science Key Lab, Beijing, China 
and Asians aged $\geq 25$ years. There was little evidence of population stratification in any of the meta-analysis results (Genomic Control lambda, $\lambda_{\mathrm{GC}}=1.014,1.011,1.018$ and 1.022 for White Europeans aged $\geq 25$ years, White European subjects aged $<25$ years, Asians aged $\geq 25$ years, and all samples combined, respectively).

Meta-analysis of White Europeans aged at least 25 years

For the meta-analysis of older White European individuals $(N=31,968)$ there were six regions containing markers with $P$ values $<5.0 \mathrm{E}-06$, suggestive of association (Table 2; Figs. 1, 2). However, only a single region contained markers that met the $P$ value conventionally accepted to declare genome-wide significance $(P<5.0 \mathrm{E}-08)$. This was at $2 \mathrm{p} 16.3$, downstream of the gene encoding neurexin-1 (NRXNI; Fig. 2a) with the most strongly associated marker being rs1401327. Each copy of the A allele of rs1401327 increased the odds of astigmatism with an OR 1.16 (95\% CI 1.10 to $1.22 ; P=3.92 \mathrm{E}-08$ ). The next most strongly associated regions were at $3 \mathrm{q} 23,4 \mathrm{p} 15,6 \mathrm{p} 22.3$, and $18 \mathrm{q} 12.1$ (Table 2). There was little evidence of heterogeneity of effect across cohorts at any of the above loci $\left(I^{2}<14\right.$; Table 2).

Meta-analysis of White Europeans aged less than 25 years

The meta-analysis of younger White European cohorts identified four regions with $P$ values below 5.0E-06 (Table 2). However, the much smaller sample size $(N=5,640)$ meant that this meta-analysis had limited statistical power to detect true-positive associations. The most strongly associated SNP was rs1366200 (OR 1.31, $95 \%$

J. B. Jonas

Department of Ophthalmology, Medical Faculty Mannheim of the Ruprecht-Karls-University of Heidelberg, Mannheim, Germany

O. Pärssinen

Department of Health Sciences and Gerontology Research

Center, University of Jyväskylä, Jyväskylä, Finland

O. Pärssinen

Department of Ophthalmology, Central Hospital of Central

Finland, Jyväskylä, Finland

D. Stambolian

University of Pennsylvania School of Medicine, Rm. 314 Stellar

Chance Labs, 422 Curie Blvd, Philadelphia, PA 19104, USA

C. J. Hammond

Department of Ophthalmology, King's College London, St

Thomas' Hospital Campus, London, UK

A. D. Paterson

Dala Lanna School of Public Health, University of Toronto,

Toronto, ON, Canada
CI $1.17-1.46 ; P=1.04 \mathrm{E}-06)$ within the AQPEP gene on chromosome $5 \mathrm{q} 23.1$.

Meta-analysis of Asians aged at least 25 years

In the meta-analysis of Asian cohorts $(N=9,295)$ the most strongly associated marker was rs7534824 (OR 2.30, $95 \%$ CI 1.65 to $3.22 ; P 9.00 \mathrm{E}-07)$ within a gene of unknown function, LOC101928334, on chromosome 1. This marker had a low allele frequency $(\mathrm{MAF}=0.03)$. Two other regions also contained SNPs with $P$ values $<5.0 \mathrm{E}-06$ (Table 2). However, this meta-analysis also had limited statistical power to detect true-positive associations.

Meta-analysis of all cohorts

To search for evidence to corroborate the initial findings, we carried out a meta-analysis of all independent individuals from the above three cohort groups combined with Asians <25 years of age from the SCORM study $(N=45,931)$. As shown in Table 3 , this revealed little evidence across cohort groups to substantiate the initial findings. The three most strongly associated regions were the NRXN1 locus, the TOX gene locus on chromosome 8q12.1, and the LINC00340 gene locus at 6p22.3, all of which were amongst the most highly associated regions identified in the meta-analysis of older White European subjects. Association at the NRXN1 gene locus (rs1401327, OR 1.139, $95 \%$ CI $1.084-1.198, P=2.93 \mathrm{E}-07$ ) was driven solely by the European cohorts, since the associated SNPs were monomorphic in Asians, and thus uninformative. The most strongly associated marker at the TOX gene locus was rs7823467 (OR 1.09, $95 \%$ CI 1.05-1.12; $P=3.47 \mathrm{E}-07$ ) while that at the LINCO0340 gene locus was rs12212674 (OR 1.09, $95 \%$ CI 1.05-1.12; $P=1.49 \mathrm{E}-06$ ).

Interestingly, the TOX region is one of the loci identified in the CREAM consortium GWAS for spherical equivalent refractive error (Verhoeven et al. 2013b) and the age of onset of myopia GWAS carried out by 23 andMe (Kiefer et al. 2013). Therefore, to investigate whether spherical refraction and astigmatism share common genetic determinants more widely, we examined the association with refractive astigmatism of 34 genome-wide significant SNPs (Table S1) reported in the CREAM (Verhoeven et al. 2013b) and 23andMe (Kiefer et al. 2013) spherical equivalent GWAS meta-analyses (4 additional SNPs associated with spherical equivalent could not be included since they were not analysed in the current study). For each SNP, the effect size (beta coefficient describing the magnitude of association) with spherical equivalent was plotted against the effect size for association with refractive astigmatism (Fig. 3). The betas were found to be highly correlated $(r=-0.59, P=2.10 \mathrm{E}-04)$. Excluding the SNP in the 
Table 1 Cohort demographics

\begin{tabular}{|c|c|c|c|c|c|c|c|}
\hline Study & Ethnicity & $N$ (cases/controls) & $\begin{array}{l}\text { Age, years } \\
(\text { mean } \pm S D)\end{array}$ & $\begin{array}{l}\text { Astigmatism, } \\
\mathrm{D}(\text { mean } \pm \mathrm{SD})\end{array}$ & $\begin{array}{l}\text { Astigmatism } \\
\text { median, D (IQR) }\end{array}$ & $\begin{array}{l}\text { Astigmatism, } \\
\text { D (range) }\end{array}$ & $\begin{array}{l}\% \\
\text { Female }\end{array}$ \\
\hline \multicolumn{8}{|l|}{ European adult cohorts } \\
\hline $\begin{array}{l}1958 \text { British } \\
\text { Birth Cohort }\end{array}$ & White European & $1,645(182 / 1,463)$ & $42 \pm 0$ & $0.47 \pm 0.53$ & $0.38(0.13-0.63)$ & $0.00-4.50$ & 45.8 \\
\hline ALSPAC mothers & White European & $1,889(343 / 1,546)$ & $44 \pm 2$ & $0.63 \pm 0.53$ & $0.50(0.25-0.75)$ & $0.00-4.62$ & 100.0 \\
\hline AREDS & White European & $1,864(567 / 1,297)$ & $68 \pm 5$ & $0.77 \pm 0.67$ & $0.75(0.25-1.00)$ & $0.00-4.50$ & 59.2 \\
\hline BATSplusTEST & White European & $204(49 / 155)$ & $40 \pm 14$ & $0.63 \pm 0.57$ & $0.38(0.25-0.89)$ & $0.00-2.75$ & 62.7 \\
\hline CROATIA-Korcula & White European & $826(135 / 691)$ & $56 \pm 13$ & $0.63 \pm 0.52$ & $0.50(0.25-0.75)$ & $0.00-4.00$ & 64.7 \\
\hline CROATIA-Split & White European & $343(35 / 308)$ & $51 \pm 13$ & $0.55 \pm 0.41$ & $0.44(0.25-0.63)$ & $0.00-3.00$ & 56.3 \\
\hline CROATIA-Vis & White European & $529(104 / 425)$ & $56 \pm 13$ & $0.68 \pm 0.57$ & $0.51(0.21-0.81)$ & $0.00-4.68$ & 59.7 \\
\hline ERF4 & White European & $2,485(472 / 2,013)$ & $49 \pm 14$ & $0.58 \pm 0.54$ & $0.50(0.25-0.75)$ & $0.00-4.13$ & 43.4 \\
\hline FITSA & White European & $87(18 / 69)$ & $68 \pm 3$ & $0.75 \pm 0.52$ & $0.63(0.38-0.88)$ & $0.00-3.50$ & 100.0 \\
\hline Framingham & White European & $1,532(745 / 787)$ & $60 \pm 12$ & $0.78 \pm 0.56$ & $0.63(0.38-1.00)$ & $0.00-4.38$ & 56.1 \\
\hline GUTENBERG & White European & $3,954(640 / 3,314)$ & $56 \pm 11$ & $0.55 \pm 0.54$ & $0.44(0.13-0.75)$ & $0.00-4.63$ & 49.2 \\
\hline KORA & White European & $1,852(448 / 1,404)$ & $56 \pm 12$ & $0.72 \pm 0.64$ & $0.50(0.25-1.00)$ & $0.00-4.75$ & 50.6 \\
\hline OGLIASTRA & White European & $472(49 / 423)$ & $52 \pm 16$ & $0.31 \pm 0.52$ & $0.00(0.00-0.50)$ & $0.00-3.00$ & 69.0 \\
\hline ORCADES & White European & $502(113 / 389)$ & $58 \pm 14$ & $0.70 \pm 0.65$ & $0.56(0.22-0.90)$ & $0.00-4.69$ & 56.8 \\
\hline ROTTERDAM 1 & White European & $5,422(2,193 / 3,229)$ & $69 \pm 9$ & $0.95 \pm 0.66$ & $0.75(0.38-1.13)$ & $0.00-4.75$ & 58.6 \\
\hline ROTTERDAM 2 & White European & $1,973(725 / 1,248)$ & $64 \pm 7$ & $0.89 \pm 0.59$ & $0.75(0.44-1.07)$ & $0.00-4.50$ & 54.3 \\
\hline ROTTERDAM 3 & White European & $1,971(580 / 1,391)$ & $56 \pm 6$ & $0.81 \pm 0.57$ & $0.63(0.31-0.94)$ & $0.00-4.00$ & 56.5 \\
\hline TwinsUK & White European & $2,658(751 / 1,907)$ & $55 \pm 13$ & $0.80 \pm 0.65$ & $0.63(0.38-1.00)$ & $0.00-4.88$ & 91.1 \\
\hline WESDR adults & White European & $280(69 / 211)$ & $35 \pm 8$ & $0.71 \pm 0.65$ & $0.50(0.19-0.81)$ & $0.00-4.50$ & 75.4 \\
\hline YFS & White European & $1,480(269 / 1,211)$ & $42 \pm 5$ & $0.64 \pm 0.52$ & $0.50(0.25-0.75)$ & $0.00-4.13$ & 55.3 \\
\hline \multicolumn{8}{|l|}{ Asian adult cohorts } \\
\hline BES & Chinese & $585(154 / 431)$ & $62 \pm 9$ & $0.66 \pm 0.59$ & $0.50(0.25-1.00)$ & $0.00-3.50$ & 65.8 \\
\hline HK-MGS adults & Chinese & $120(59 / 61)$ & $34 \pm 7$ & $1.29 \pm 1.05$ & $0.97(0.50-1.84)$ & $0.00-5.31$ & 61.7 \\
\hline SCES & Chinese & $1,662(670 / 992)$ & $57 \pm 9$ & $0.99 \pm 0.63$ & $0.85(0.48-1.23)$ & $0.00-4.30$ & 48.8 \\
\hline SIMES & Malay & $2,165(706 / 1,459)$ & $57 \pm 11$ & $0.90 \pm 0.66$ & $0.73(0.39-1.06)$ & $0.00-4.85$ & 50.8 \\
\hline SINDI & Indian & $1,998(739 / 1,259)$ & $56 \pm 9$ & $0.96 \pm 0.62$ & $0.83(0.47-1.18)$ & $0.00-4.53$ & 48.7 \\
\hline SP2 & Chinese & $1,954(543 / 1,411)$ & $48 \pm 11$ & $0.81 \pm 0.56$ & $0.68(0.36-0.99)$ & $0.00-4.18$ & 54.2 \\
\hline STARS & Chinese & $811(205 / 606)$ & $39 \pm 5$ & $0.72 \pm 0.67$ & $0.60(0.21-0.94)$ & $0.00-4.32$ & 48.0 \\
\hline \multicolumn{8}{|c|}{ European youngsters cohorts } \\
\hline ALSPAC children & White European & $3,828(580 / 3,248)$ & $15 \pm 0.3$ & $0.65 \pm 0.42$ & $0.63(0.38-0.75)$ & $0.00-4.25$ & 48.8 \\
\hline $\begin{array}{l}\text { BATSplusTEST } \\
\text { children }\end{array}$ & White European & $561(60 / 501)$ & $18 \pm 4$ & $0.40 \pm 0.48$ & $0.25(0.13-0.5)$ & $0.00-4.00$ & 54.0 \\
\hline RAINE & White European & $1,007(215 / 792)$ & $20 \pm 0$ & $0.74 \pm 0.40$ & $0.69(0.45-0.93)$ & $0.08-3.11$ & 49.3 \\
\hline WESDR children & White European & $244(52 / 192)$ & $18 \pm 4$ & $0.64 \pm 0.57$ & $0.50(0.25-0.75)$ & $0.00-3.38)$ & 50.8 \\
\hline \multicolumn{8}{|l|}{ Asian youngsters cohort } \\
\hline SCORM & Chinese & $917(247 / 670)$ & $11 \pm 1$ & $0.77 \pm 0.66$ & $0.57(0.21-0.94)$ & $0.00-4.32$ & 48.0 \\
\hline
\end{tabular}

TOX gene region had minimal influence on the correlation of the betas for the remaining 33 SNPs $(r=-0.60$, $P=2.29 \mathrm{E}-04)$.

\section{Discussion}

This GWAS meta-analysis of nearly 46,000 individuals identified several novel, suggestive candidate genes/ regions for refractive astigmatism, including $N R X N 1$, TOX and LINC00340. One of these regions, near the NRXN1 gene region, reached genome-wide significance in the White European adult group. Two-thirds of the $\sim 46,000$ subjects included in the full meta-analysis were White European adults and so the results are likely to have been driven mainly by this group. Therefore, until the opportunity arises for replication in independent samples, especially in large numbers of comparable 
Table 2 Most strongly associated SNPs in the 3 meta-analyses

\begin{tabular}{|c|c|c|c|c|c|c|c|c|c|c|c|}
\hline SNP & $\mathrm{Chr}$ & Pos & RA & NRA & RAF (min-max) & OR & $95 \% \mathrm{CI}$ & $P$ value & $I^{2}$ & $N$ & Gene(s) \\
\hline \multicolumn{12}{|c|}{ European subjects aged $\geq 25$ years } \\
\hline rs 1401327 & 2 & 49900987 & A & G & $0.113-0.174$ & 1.157 & $1.098-1.218$ & $3.92 \mathrm{E}-08$ & 0 & 31,694 & $N R X N 1$ \\
\hline rs17795388 & 2 & 49900356 & $\mathrm{G}$ & A & $0.113-0.174$ & 1.157 & $1.098-1.218$ & $4.16 \mathrm{E}-08$ & 0 & 31,691 & $N R X N 1$ \\
\hline rs11690625 & 2 & 49908115 & $\mathrm{C}$ & A & $0.113-0.175$ & 1.156 & $1.098-1.218$ & $4.17 \mathrm{E}-08$ & 0 & 31,731 & $N R X N 1$ \\
\hline rs17795358 & 2 & 49897928 & A & G & $0.113-0.173$ & 1.156 & $1.097-1.218$ & $4.94 \mathrm{E}-08$ & 0 & 31,672 & $N R X N 1$ \\
\hline rs925931 & 2 & 49913312 & $\mathrm{C}$ & $\mathrm{T}$ & $0.113-0.173$ & 1.148 & $1.090-1.210$ & $2.06 \mathrm{E}-07$ & 0 & 31,727 & $N R X N 1$ \\
\hline rs 885560 & 2 & 49909442 & G & A & $0.113-0.175$ & 1.146 & $1.088-1.207$ & $2.46 \mathrm{E}-07$ & 0 & 31,728 & $N R X N 1$ \\
\hline rs6708111 & 2 & 49878453 & A & $\mathrm{C}$ & $0.102-0.168$ & 1.139 & $1.082-1.200$ & $7.27 \mathrm{E}-07$ & 0 & 31,531 & $N R X N 1$ \\
\hline rs11690252 & 2 & 49890187 & $\mathrm{~T}$ & $\mathrm{G}$ & $0.230-0.342$ & 1.105 & $1.060-1.151$ & $2.59 \mathrm{E}-06$ & 0 & 31,511 & $N R X N 1$ \\
\hline rs1878856 & 2 & 49877706 & $\mathrm{C}$ & $\mathrm{T}$ & $0.214-0.336$ & 1.105 & $1.059-1.153$ & $3.56 \mathrm{E}-06$ & 0 & 31,603 & $N R X N 1$ \\
\hline rs12638075 & 3 & $1.42 \mathrm{E}+08$ & $\mathrm{C}$ & $\mathrm{T}$ & $0.014-0.024$ & 1.376 & $1.200-1.577$ & $4.69 \mathrm{E}-06$ & 0 & 27,304 & TRIM42/CLSTN2 \\
\hline rs2309717 & 4 & 27859336 & A & $\mathrm{C}$ & $0.089-0.170$ & 1.143 & $1.083-1.206$ & $1.02 \mathrm{E}-06$ & 11.8 & 31,143 & STIM2/PCDH7 \\
\hline rs2871434 & 4 & 29931147 & $\mathrm{~T}$ & A & $0.095-0.154$ & 1.140 & 1.079-1.204 & $2.66 \mathrm{E}-06$ & 13.6 & 31,664 & STIM2/PCDH7 \\
\hline rs 12212674 & 6 & 22195053 & A & $\mathrm{T}$ & $0.496-0.621$ & 1.099 & $1.058-1.142$ & $1.45 \mathrm{E}-06$ & 0 & 31,691 & LINC00340 \\
\hline rs6901423 & 6 & 22194271 & G & A & $0.496-0.621$ & 1.099 & $1.057-1.142$ & $1.63 \mathrm{E}-06$ & 0 & 31,689 & LINC00340 \\
\hline rs4712652 & 6 & 22186594 & A & G & $0.495-0.687$ & 1.097 & $1.055-1.141$ & $3.13 \mathrm{E}-06$ & 0 & 28,910 & LINC00340 \\
\hline rs9366427 & 6 & 22204592 & G & $\mathrm{C}$ & $0.487-0.619$ & 1.094 & $1.053-1.136$ & $4.15 \mathrm{E}-06$ & 0 & 31,773 & LINC00340 \\
\hline rs4799964 & 18 & 26239477 & G & $\mathrm{T}$ & $0.020-0.048$ & 1.267 & $1.152-1.394$ & $1.16 \mathrm{E}-06$ & 0 & 31,881 & $M I R 302 F$ \\
\hline rs12607243 & 18 & 26229228 & G & A & $0.020-0.050$ & 1.264 & $1.149-1.392$ & $1.60 \mathrm{E}-06$ & 0 & 31,882 & MIR302F \\
\hline \multicolumn{12}{|c|}{ European subjects aged $<25$ years } \\
\hline rs6688613 & 1 & 165218493 & $\mathrm{~T}$ & $\mathrm{C}$ & $0.240-0.253$ & 1.309 & $1.170-1.465$ & $2.68 \mathrm{E}-06$ & 0 & 5,640 & $M A E L$ \\
\hline rs 1327866 & 1 & 165219534 & G & A & $0.238-0.253$ & 1.308 & $1.169-1.464$ & $2.89 \mathrm{E}-06$ & 0 & 5,640 & $M A E L$ \\
\hline rs7550698 & 1 & 165217705 & $\mathrm{C}$ & $\mathrm{T}$ & $0.240-0.253$ & 1.308 & $1.168-1.463$ & $3.02 \mathrm{E}-06$ & 0 & 5,640 & $M A E L$ \\
\hline rs7528849 & 1 & 165221494 & G & A & $0.240-0.253$ & 1.307 & $1.168-1.463$ & $3.11 \mathrm{E}-06$ & 0 & 5,640 & $M A E L$ \\
\hline rs7518155 & 1 & 165221520 & G & $\mathrm{T}$ & $0.240-0.253$ & 1.307 & $1.168-1.462$ & $3.19 \mathrm{E}-06$ & 0 & 5,640 & $M A E L$ \\
\hline rs7545911 & 1 & 165214305 & A & G & $0.240-0.253$ & 1.309 & $1.169-1.467$ & $3.35 \mathrm{E}-06$ & 0 & 5,640 & $M A E L$ \\
\hline rs6682062 & 1 & 165216603 & $\mathrm{C}$ & G & $0.240-0.253$ & 1.309 & $1.168-1.467$ & $3.39 \mathrm{E}-06$ & 0 & 5,640 & $M A E L$ \\
\hline rs2296837 & 1 & 165225225 & $\mathrm{C}$ & $\mathrm{T}$ & $0.240-0.253$ & 1.305 & $1.166-1.461$ & $3.53 \mathrm{E}-06$ & 0 & 5,640 & $M A E L$ \\
\hline rs11578336 & 1 & 165225334 & G & $\mathrm{T}$ & $0.240-0.253$ & 1.304 & $1.166-1.460$ & $3.71 \mathrm{E}-06$ & 0 & 5,640 & $M A E L$ \\
\hline rs 1366200 & 5 & 115349718 & G & $\mathrm{T}$ & $0.312-0.321$ & 1.308 & $1.174-1.457$ & $1.04 \mathrm{E}-06$ & 48.7 & 5,640 & $A Q P E P$ \\
\hline rs17712049 & 7 & 48236741 & $\mathrm{C}$ & $\mathrm{T}$ & $0.875-0.904$ & 1.569 & $1.295-1.902$ & $4.39 \mathrm{E}-06$ & 0 & 5,640 & $A B C A 13$ \\
\hline rs13257518 & 8 & 32755116 & A & $\mathrm{T}$ & $0.177-0.217$ & 1.370 & $1.202-1.561$ & $2.36 \mathrm{E}-06$ & 15.7 & 5,640 & $N R G 1$ \\
\hline rs10503929 & 8 & 32733525 & $\mathrm{C}$ & $\mathrm{T}$ & $0.167-0.215$ & 1.352 & $1.192-1.534$ & $2.68 \mathrm{E}-06$ & 32.3 & 5,640 & $N R G 1$ \\
\hline rs 2975500 & 8 & 32724907 & A & G & $0.110-0.161$ & 1.435 & $1.231-1.673$ & $3.95 \mathrm{E}-06$ & 0 & 5,640 & $N R G 1$ \\
\hline \multicolumn{12}{|l|}{ Asian adults } \\
\hline rs7534824 & 1 & 101394034 & A & G & $0.967-0.974$ & 2.304 & $1.651-3.214$ & $9.00 \mathrm{E}-07$ & 0 & 4,812 & LOC101928334 \\
\hline rs10496034 & 2 & 54998439 & $\mathrm{C}$ & G & $0.170-0.287$ & 1.216 & $1.122-1.318$ & $2.13 \mathrm{E}-06$ & 0 & 8,780 & EML6 \\
\hline rs428445 & 20 & 54469954 & $\mathrm{~T}$ & G & $0.713-0.954$ & 1.314 & $1.175-1.470$ & $1.84 \mathrm{E}-06$ & 0 & 8,908 & CASS4/GCNT7 \\
\hline rs6999 & 20 & 54527308 & A & G & $0.713-0.957$ & 1.303 & $1.164-1.459$ & $4.30 \mathrm{E}-06$ & 0 & 8,904 & CASS4/GCNT7 \\
\hline
\end{tabular}

The table shows all SNPs with $P<5.0 \mathrm{E}-06$

$R A$ risk allele, $N R A$ non-risk (reference) allele, $R A F$ risk allele frequency in each cohort, $O R$ odds ratio, $95 \% C I 95 \%$ confidence interval of odds ratio, $I^{2}$ heterogeneity statistic, $N$ total sample size

White European adults, caution is needed in interpreting these results. These results should not be considered to be relevant to other populations until replicated in younger White European samples or in other ethnic groups.
Novel candidate genes underlying the observed associations

Neurexin-1, one of the largest genes in the human genome, is thought to function in cell adhesion, as well as synapse 
Table 3 Most strongly associated SNPs in the meta-analysis of all cohorts

\begin{tabular}{|c|c|c|c|c|c|c|c|c|c|c|c|}
\hline SNP & $\mathrm{Chr}$ & Pos & RA & NRA & RAF (min-max) & OR & $95 \% \mathrm{CI}$ & $P$ value & $I^{2}$ & $N$ & Gene \\
\hline rs1401327 & 2 & 49900987 & A & G & $0.113-0.174$ & 1.139 & $1.084-1.198$ & $2.93 \mathrm{E}-07$ & 0 & 35,445 & NRXN1 \\
\hline rs11690625 & 2 & 49908115 & $\mathrm{C}$ & A & $0.113-0.175$ & 1.139 & $1.084-1.197$ & $2.95 \mathrm{E}-07$ & 0 & 35,482 & NRXN1 \\
\hline rs 17795388 & 2 & 49900356 & $\mathrm{G}$ & A & $0.113-0.174$ & 1.139 & $1.084-1.198$ & $3.10 \mathrm{E}-07$ & 0 & 35,442 & NRXN1 \\
\hline rs 17795358 & 2 & 49897928 & A & $\mathrm{G}$ & $0.113-0.173$ & 1.139 & $1.083-1.197$ & $3.67 \mathrm{E}-07$ & 0 & 35,423 & NRXN1 \\
\hline rs925931 & 2 & 49913312 & $\mathrm{C}$ & $\mathrm{T}$ & $0.010-0.173$ & 1.125 & $1.071-1.182$ & $2.64 \mathrm{E}-06$ & 3.3 & 39,567 & NRXN1 \\
\hline rs885560 & 2 & 49909442 & G & A & $0.010-0.175$ & 1.123 & $1.069-1.179$ & $3.46 \mathrm{E}-06$ & 5.5 & 39,566 & NRXN1 \\
\hline rs6708111 & 2 & 49878453 & A & $\mathrm{C}$ & $0.102-0.168$ & 1.124 & $1.069-1.182$ & $4.42 \mathrm{E}-06$ & 0 & 35,282 & NRXN1 \\
\hline rs7581641 & 2 & 8543557 & $\mathrm{~T}$ & $\mathrm{C}$ & $0.012-0.103$ & 1.225 & $1.123-1.336$ & $4.74 \mathrm{E}-06$ & 0 & 41,865 & NRXN1 \\
\hline rs6892230 & 5 & 65175520 & A & G & $0.016-0.078$ & 1.236 & $1.133-1.349$ & $1.87 \mathrm{E}-06$ & 41.3 & 37,591 & NLN \\
\hline rs 12212674 & 6 & 22195053 & A & $\mathrm{T}$ & $0.134-0.621$ & 1.086 & $1.050-1.123$ & $1.49 \mathrm{E}-06$ & 0 & 45,134 & LINC00340 \\
\hline rs6901423 & 6 & 22194271 & G & A & $0.134-0.621$ & 1.083 & $1.048-1.120$ & $3.00 \mathrm{E}-06$ & 0 & 45,132 & LINC00340 \\
\hline rs1034071 & 6 & 22205354 & $\mathrm{C}$ & $\mathrm{T}$ & $0.137-0.608$ & 1.081 & $1.046-1.118$ & $3.73 \mathrm{E}-06$ & 0 & 45,330 & LINC00340 \\
\hline rs7823467 & 8 & 60241288 & $\mathrm{~T}$ & $\mathrm{C}$ & $0.388-0.713$ & 1.085 & $1.052-1.120$ & $3.47 \mathrm{E}-07$ & 22.9 & 45,273 & TOX \\
\hline rs10086929 & 8 & 60252851 & A & G & $0.430-0.709$ & 1.083 & $1.049-1.118$ & $7.36 \mathrm{E}-07$ & 22.3 & 45,156 & TOX \\
\hline rs6471768 & 8 & 60230697 & $\mathrm{~T}$ & A & $0.435-0.710$ & 1.082 & $1.048-1.117$ & $1.07 \mathrm{E}-06$ & 23.9 & 45,125 & TOX \\
\hline rs4531042 & 8 & 60251242 & G & A & $0.388-0.737$ & 1.082 & $1.048-1.118$ & $1.45 \mathrm{E}-06$ & 32.9 & 45,277 & TOX \\
\hline rs4738757 & 8 & 60218783 & A & G & $0.388-0.701$ & 1.080 & $1.046-1.115$ & $1.89 \mathrm{E}-06$ & 26.9 & 45,122 & TOX \\
\hline rs 12675886 & 8 & 60309643 & $\mathrm{C}$ & $\mathrm{T}$ & $0.458-0.704$ & 1.079 & $1.045-1.114$ & $2.50 \mathrm{E}-06$ & 14.3 & 45,082 & TOX \\
\hline rs6997378 & 8 & 60330443 & $\mathrm{~T}$ & G & $0.460-0.705$ & 1.077 & $1.043-1.111$ & $4.95 \mathrm{E}-06$ & 17.3 & 45,085 & TOX \\
\hline rs1944146 & 11 & 130195372 & A & G & $0.524-0.608$ & 1.080 & $1.046-1.115$ & $2.62 \mathrm{E}-06$ & 17.3 & 45,243 & LOC100507431 \\
\hline rs7934985 & 11 & 130194532 & $\mathrm{G}$ & A & $0.523-0.613$ & 1.080 & $1.046-1.116$ & $2.66 \mathrm{E}-06$ & 4.8 & 45,123 & LOC100507431 \\
\hline
\end{tabular}

The table shows all SNPs with $P<5.0 \mathrm{E}-06$
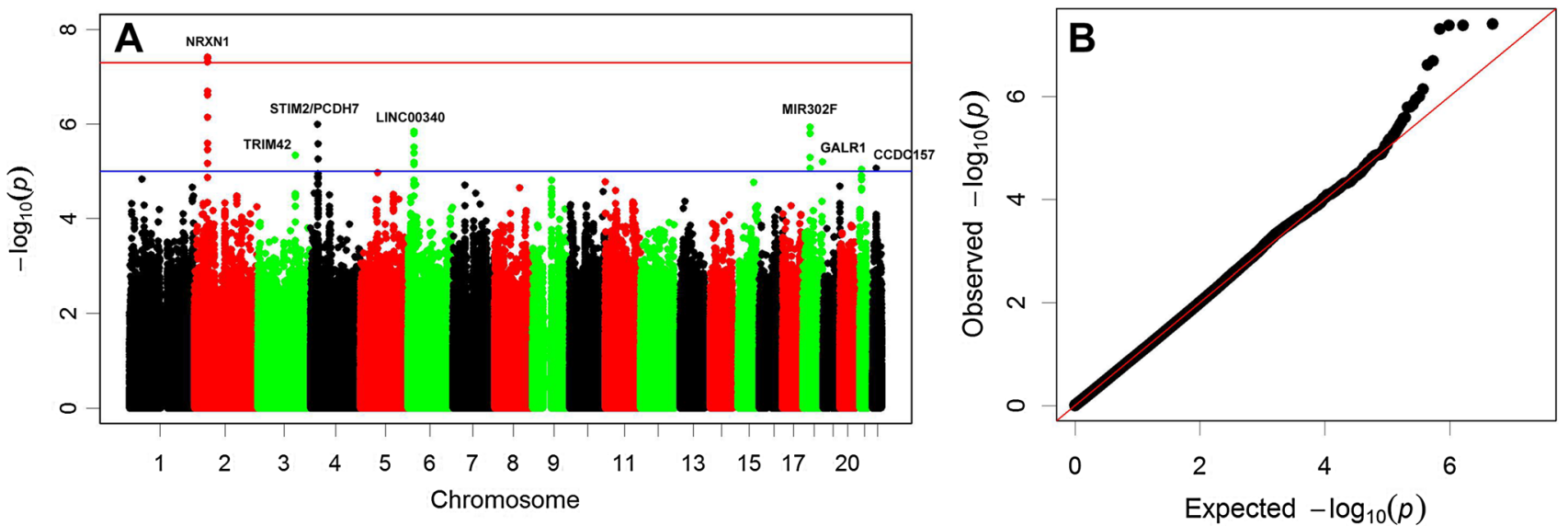

Fig. 1 Results of the meta-analysis of White European subjects aged $\geq 25$ years old. a Manhattan plot of $\log P$ values against genomic position. The red horizontal line is the threshold commonly used to for declaring genome-wide significance $(P=5.0 \mathrm{E}-08)$. The blue

development and maintenance (Kirov et al. 2008, 2009). Structural genomic deletions that delete or disrupt NXRN1 are strongly implicated in causing psychiatric and cognitive phenotypes including schizophrenia, autism and mental retardation (Bena et al. 2013). To our knowledge, these conditions are not known to be associated with refractive line indicates $P=1.0 \mathrm{E}-05$. Genes adjacent to the association signal are indicated. b Quantile-quantile (QQ) plot of observed versus expected distribution of $\log P$ values. The red line shows the distribution expected by chance

astigmatism (although refractive errors, in general, are more prevalent in individuals with learning difficulties, Woodhouse et al. 2003). A recent survey of 25 patients with exonic deletions involving the gene for neurexin-1 (Bena et al. 2013) unfortunately did not describe these patients' ocular features. While the strength of association reached genome-wide 

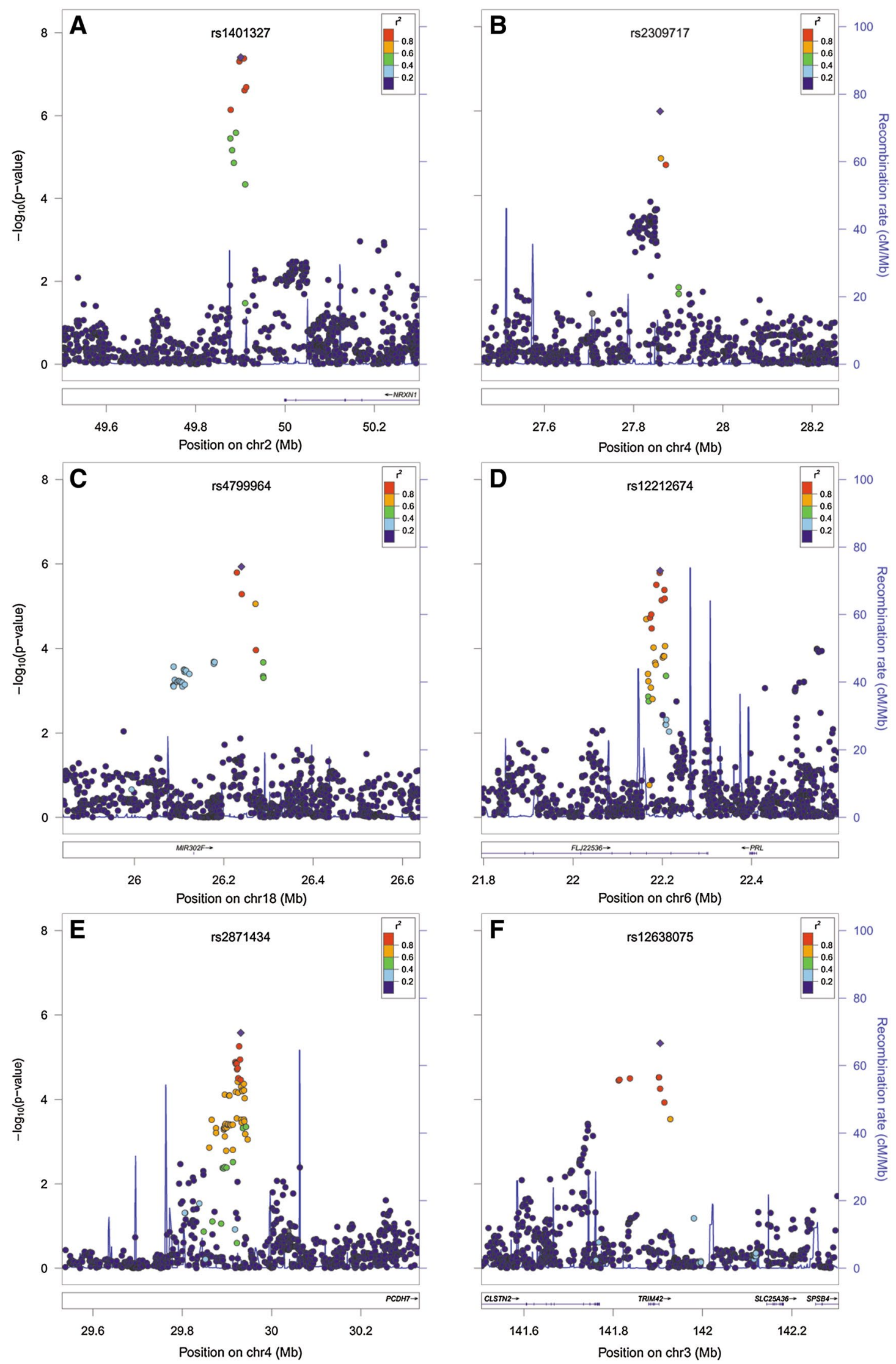

Fig. 2 Regions showing the strongest evidence for association with refractive astigmatism in the meta-analysis of White Europeans aged $\geq 25$ years 
Fig. 3 Common genetic determinants for spherical equivalent refractive error are shared with refractive astigmatism. GWAS meta-analysis beta coefficients (which quantify the effect size of SNPs) were compared between studies of spherical equivalent and refractive astigmatism. The SNP beta coefficients for spherical equivalent were obtained from the CREAM consortium GWAS for spherical equivalent (Verhoeven et al. 2013b), while those for refractive astigmatism were from the current study. The 34 SNPs analysed were chosen based on prior genome-wide significant evidence of association with spherical equivalent in the CREAM (Verhoeven et al. 2013b) and 23andMe (Kiefer et al. 2013) GWAS meta-analyses. The solid line is the best linear fit to the data

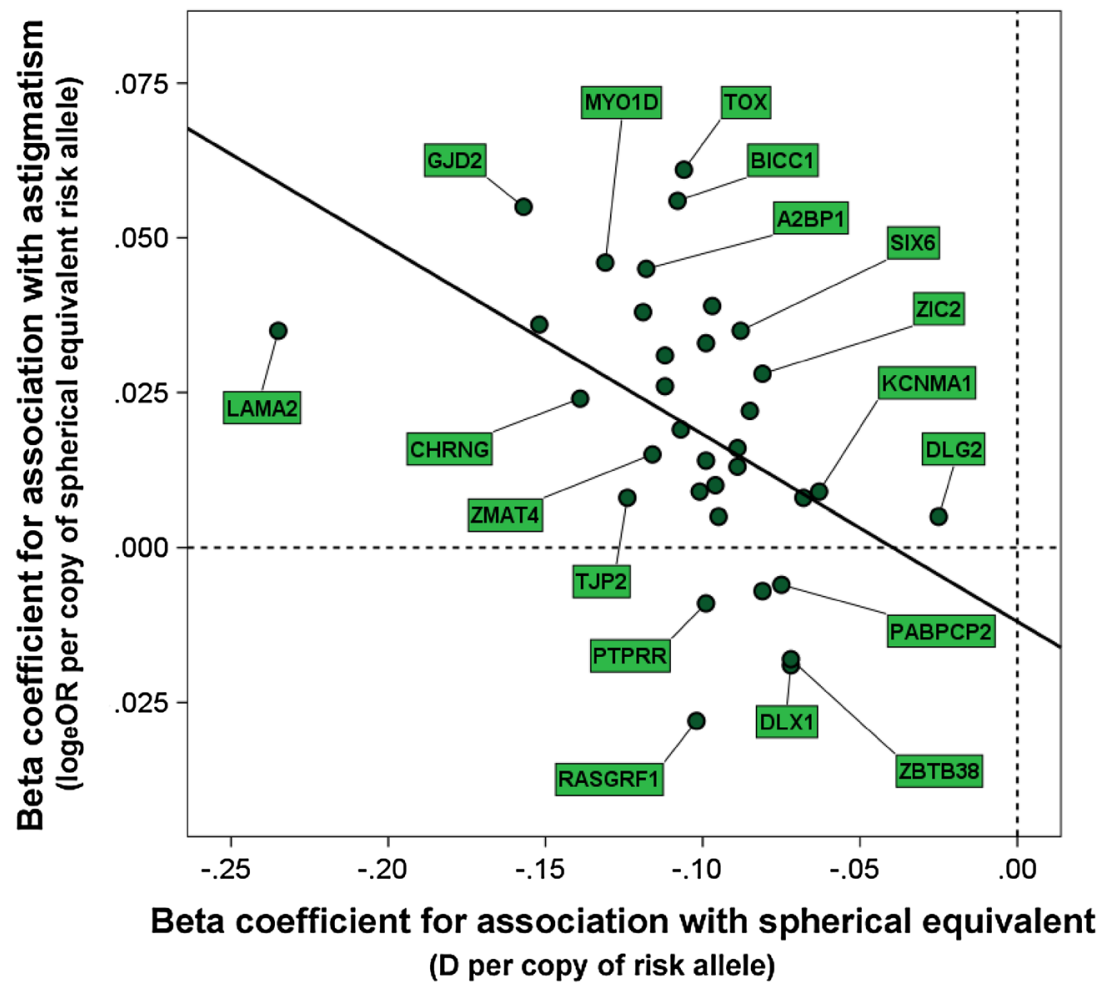

significance in the adult European sample $(N=31,968$, $P=3.92 \mathrm{E}-08)$, this weakened when the younger European subjects were included $(N=35,719, P=2.93 \mathrm{E}-07)$ while having little impact on the estimated effect size (OR 1.16 and 1.14 , respectively). The associated SNPs in this region were monomorphic in Asian subjects, suggesting they arose relatively recently in human evolution.

The associated variants at $8 \mathrm{q} 12.1$ lie upstream of the TOX promoter and thus would be well placed to influence its transcription level. However, it is not clear whether TOX or a nearby gene mediates this locus' impact on spherical equivalent refractive error, and potentially astigmatism. The known roles of TOX relate to immune function, which argues against a role in refractive development and instead suggests that another gene such as SDCBP (syndecanbinding protein) also known as syntenin, which lies $600 \mathrm{~kb}$ from the most strongly associated marker may be involved. Syntenin acts as a link between the proteoglycan/matrix receptor syndecan-1 and the cytoskeleton, and its proposed functions include cell adhesion. Furthermore, synteninnull mice show wound healing defects that are particularly marked in the cornea (Stepp et al. 2002, 2010).

The 6p22.3 locus containing the long intergenic non-coding RNA gene LINC00340 (also known as FLJ22536 and CASC15) is gene poor (Fig. 2d) yet has previously shown association with aggressive neuroblastoma in GWAS studies (Capasso et al. 2013). The mechanisms through which non-coding RNAs act are poorly understood (Guttman et al. 2009; Gibb et al. 2011) but in the case of lincRNAs the mechanism may involve epigenetic regulation (Salta and De Strooper 2012). No obvious candidate astigmatism susceptibility gene is present in this genomic location. As with $N R X N 1$, the association with LINC00340 was almost wholly driven by the adult European cohorts $(P=1.45 \mathrm{E}-06$ versus $P=1.49 \mathrm{E}-06$ in all cohorts combined).

As well as NRXNI and SDCBP, additional genes in the most strongly associated regions have putative roles in cell adhesion and/or synapse function. The gene nearest to the lead SNP at 3q23 in European adults (rs12638075, $P=4.69 \mathrm{E}-06$ ) is TRIM42 (tripartite motif containing-42). Because members of the TRIM gene family function mostly in immune signalling (Versteeg Gijs et al. 2013), the adjacent gene CLSTN2 (calsyntenin-2; also known as cadherinrelated family member-13) is potentially of greater interest given its proposed role in cell adhesion and synapse function (Preuschhof et al. 2010). Furthermore, the association described above for markers in the vicinity of the SDCBP gene, encoding syntenin, lends support to the putative involvement of CLSTN2. One of the two regions on chromosome 4p15 (lead SNP rs2871434; Fig. 2e) contains the $\mathrm{PCDH7}$ (protocadherin-7) gene, which given its role in cell adhesion is a plausible candidate gene for astigmatism. In mice homozygous for a null allele of the EGRI gene, which develop a transient axial myopia postnatally, a member 
Table 4 Results from the meta-analysis of all cohorts for SNPs previously associated with corneal astigmatism (CA) or refractive astigmatism (RA)

\begin{tabular}{lllllllllllll}
\hline Trait & SNP & Chr & RA & NRA & RAF (min-max) & OR & $95 \%$ CI & $P$ value & $I^{2}$ & $N$ & Gene & References \\
\hline RA & rs3771395 & 2 & A & G & $0.06-0.30$ & 1.04 & $1.00-1.09$ & $5.17 \mathrm{E}-02$ & 19.2 & 45,324 & VAX2 & Lopes et al. (2013) \\
CA & rs7677751 & 4 & T & C & $0.07-0.26$ & 1.03 & $0.99-1.08$ & $1.03 \mathrm{E}-01$ & 17.9 & 45,287 & $P D G F R A$ & Fan et al. (2011) \\
RA & rs795544 & 5 & C & A & $0.64-0.92$ & 1.05 & $1.01-1.09$ & $2.01 \mathrm{E}-02$ & 0 & 45,245 & DNAH5 & Lopes et al. (2013) \\
RA & rs10226930 & & & & & & & & & & SHH & Lopes et al. (2013) \\
RA & rs485842 & 11 & C & T & $0.33-0.77$ & 1.05 & $1.01-1.08$ & $1.21 \mathrm{E}-02$ & 10.2 & 45,137 & MAML2 & Lopes et al. (2013) \\
RA & rs12445126 & 16 & A & G & $0.02-0.14$ & 1.02 & $0.97-1.09$ & $4.16 \mathrm{E}-01$ & 21.1 & 45,198 & $X Y L T 1$ & Lopes et al. (2013) \\
RA & rs11644988 & 16 & G & A & $0.73-0.99$ & 1.04 & $0.98-1.11$ & $2.46 \mathrm{E}-01$ & 0 & 40,369 & FOXF1 & Lopes et al. (2013) \\
\hline
\end{tabular}

${ }^{\text {a }}$ SNP not present in current meta-analysis

of the protocadherin gene family, Pcdhb9, was the most highly differentially expressed retinal gene when compared to wild-type mice (Schippert et al. 2009). The second associated region at 4p15 (lead SNP rs2309717; Fig. 2b) contains no known genes, the closest being MIR4275, which lies $600 \mathrm{~kb}$ away. However, amongst the more than 6,000 predicted targets of miR-4275 is the nearby $P C D H 7$.

Genetic co-determination of spherical equivalent and refractive astigmatism

One of the most exciting findings from this study was the evidence for overlap in genetic susceptibility between spherical and astigmatic refractive errors (Fig. 3). It is well known that spherical and astigmatic refractive errors tend to co-occur (Read et al. 2007; Guggenheim and Farbrother 2004). However, to our knowledge this is the first study to provide evidence supporting shared genetic susceptibility for the two traits. Kee and Deng (2008) and Kee et al. (2005) have shown in monkeys and chickens that visual experience can alter spherical equivalent and astigmatic refractive errors concurrently. Hence, in line with the view that genetic factors might alter refractive development by regulating how the eye responds to visual cues (Chen et al. 2011; Wallman 1994), it is feasible that causal variants tagged by the SNPs examined here impact on both spherical equivalent and astigmatism via visual feedback.

The suggestive findings here that genes related to cell adhesion and synapse function may be involved in susceptibility to astigmatism is also consistent with the concept of genetic co-determination of spherical equivalent and refractive astigmatism, as several candidate genes identified in GWAS for spherical equivalent refractive error have putative roles in synapse function or plasticity, for example RASGRF1, GRIA4, RBFOX1, LRRC4C, DLG2 (Kiefer et al. 2013; Verhoeven et al. 2013b; Stambolian et al. 2013; Hysi et al. 2010) as well as in cell adhesion, for example TJP2, CTNND2, ANTXR2, and LRFN5 (Kiefer et al. 2013; Li et al. 2011; Verhoeven et al. 2013b).
Comparison with previous work and limitations of the current study

Results from the meta-analysis of all cohorts for SNPs previously associated with astigmatism are shown in Table 4. Because the cohorts studied here overlap substantially with those examined previously (Fan et al. 2011; Lopes et al. 2013), low $P$ values were expected-but not found. Thus the $P$ values in Table 4 provide little evidence for replication of the previously associated markers. This is especially surprising for the corneal astigmatism-associated SNP at the PDGFRA locus (Fan et al. 2011), since this has already been replicated in a cohort of differing ethnicity (Guggenheim et al. 2013). Instead, the lack of replication may reflect the different traits examined (corneal versus refractive astigmatism). The other SNPs previously associated with astigmatism did not reach genome-wide significance in the original study, and were associated with astigmatism when analysed as a quantitative trait, which may explain the lack of independent replication.

Genetic studies of astigmatism are hampered by the variation in its magnitude and orientation with age, and its nonGaussian frequency distribution, all of which complicate the choice of analysis model. In younger individuals, astigmatism is typically "with the rule" (WTR; axis of minus power cylindrical correcting lens close to horizontal) while in later life it usually switches to "against the rule" (ATR; correcting negative cylinder axis close to vertical) (Mandel et al. 2010; Guggenheim and Farbrother 2004). Amongst the theories explaining this transition, a loosening of eyelid tension is the most widely supported (Read et al. 2007). If it is the case that ATR and WTR astigmatism have different etiologies, then GWAS investigations should attain maximum statistical power by modelling younger and older subjects separately, modelling ATR and WTR astigmatism separately, or in modelling astigmatism as a vector quantity. However, the age-dependent shift in WTR to ATR largely concerns low-level astigmatism whereas higher levels may be more stable over the life course (Baldwin and 
Mills 1981; Weale 2003). Thus, the present study adopted a dichotomous case/control classification scheme, and analysed younger and older subjects separately, in an attempt to mitigate the effects of axis changes with age. The dichotomization scheme also allayed concerns regarding the nonnormality of the trait, although this would have been at the expense of statistical power.

The use of a dichotomous phenotype definition for our GWAS meta-analysis of astigmatism contrasts with the quantitative trait approach used in previous GWAS metaanalyses by the CREAM consortium for refractive error and axial eye length (Verhoeven et al. 2013b; Cheng et al. 2013). It has been shown that binary trait GWAS metaanalysis results are sensitive to unequal numbers of cases and controls in individual cohorts, especially when the sample size is small (Willer et al. 2010). However, we found very similar results when overcoming this potential source of bias using an "effective sample size" rather than actual sample size during meta-analysis (Willer et al. 2010). In addition to the problem of unequal case/control sample sizes, we also observed highly inflated type-I errors during initial meta-analysis trials due to extreme OR estimates for a small number of low MAF markers in certain cohorts, e.g. if the minor allele was present in controls but absent in cases. To circumvent this, we pre-screened each GWAS results file, excluding markers with unfeasibly high OR estimates $(\mathrm{OR}<0.2$ or $\mathrm{OR}>5.0)$.

Out of 7 Asian adult cohorts (total $N=9,295$ ), 5 were Chinese cohorts $(N=5,132$, about $55 \%$ of the total Asian adult sample). Therefore, we cannot generalise our results from the Asian adult group with ease. Importantly, the SNP (rs7534824, in the gene LOC101928334) which showed the strongest suggestive association in the Asian group was only polymorphic in the Chinese cohorts (monomorphic in the Indian and Malay cohorts). For the other 3 SNPs reported in Table 2, although they are polymorphic in all three ethnic groups, the association signal was mainly driven by the observed association in the 5 Chinese cohorts.

In summary, this large-scale meta-analysis of GWAS studies for refractive astigmatism identified only a single locus that reached genome-wide significance ( 2 p16.3, near $N R X N 1$, in European adults) and there was no evidence for replication of this region in younger European individuals or in non-Europeans. Several putative candidate genes with functions relating to cell adhesion and/or synapse function were present in the next most strongly associated regions. Consistent with earlier work, all of the most strongly associated genetic variants identified had small effects, supporting the polygenic nature of genetic susceptibility to refractive astigmatism in the general population. Fewer candidate risk variants were discovered for refractive astigmatism than were found previously by the CREAM consortium for spherical equivalent refractive error (Verhoeven et al. 2013b), despite studying similar subject cohorts. Nevertheless, there was compelling evidence for shared genetic susceptibility for spherical and astigmatic refractive errors, implying that the co-occurrence of these traits is, at least in part, genetically determined.

Conflict of interest None of the authors have any conflicts of interest regarding the work reported here.

Open Access This article is distributed under the terms of the Creative Commons Attribution License which permits any use, distribution, and reproduction in any medium, provided the original author(s) and the source are credited.

\section{Appendix}

The CREAM consortium Akira Meguro, Alan F. Wright, Alex W. Hewitt, Alvin L. Young, Amutha Barathi Veluchamy, Andres Metspalu, Andrew D. Paterson, Angela Döring, Anthony P. Khawaja, Barbara E. Klein, Beate St Pourcain, Ben A. Oostra, Brian Fleck, Caroline C. Klaver, Caroline Hayward, Cathy Williams, Cécile Delcourt, Cecilia Maubaret, Chi Pui Pang, Chiea-Chuen Khor, Ching-Yu Cheng, Christian Gieger, Christopher J. Hammond, Claire L. Simpson, Cornelia M. van Duijn, Daniel W. H. Ho, David A. Mackey, David M. Evans, Dwight Stambolian, Emily Chew, E-Shyong Tai, Evelin Mihailov, Federico Murgia, George Davey Smith, George McMahon, Ginevra Biino, Harry Campbell, Igor Rudan, Ilkka Seppala, Jaakko Kaprio, James F. Wilson, Jamie E. Craig, Jan Roelof Polling, Janina S. Ried, Jan-Willem Tideman, Jeremy A. Guggenheim, Jeremy R. Fondran, Jie Jin Wang, Jiemin Liao, Jing Hua Zhao, Jing Xie, Joan E. Bailey Wilson, John P. Kemp, Jost B. Jonas, Jugnoo S. Rahi, Juho Wedenoja, Kari-Matti Mäkelä, Kathryn P. Burdon, KayTee Khaw, Kenji Yamashiro, Konrad Oexle, Laura Portas, Lindsay Farrer, Leslie J. Raffel, Li Jia Chen, Liang Xu, M. Kamran Ikram, Margaret M. Deangelis, Margaux Morrison, Maria Schache, Mario Pirastu, Mary-Frances Cotch, Masahiro Miyake, Maurice K.H. Yap, Maurizio Fossarello, Mika Kähönen, Mingguang He, Nagahisa Yoshimura, Nicholas G. Martin, Nicholas J. Timpson, Nick J. Wareham, Nobuhisa Mizuki, Norbert Pfeiffer, Olavi Pärssinen, Olli Raitakari, Ozren Polasek, Pancy O. Tam, Paul J. Foster, Paul Mitchell, Paul N. Baird, Peng Chen, Pirro G. Hysi, Puya Gharahkhani, Qiao Fan, René Höhn, Rhys D. Fogarty, Robert N. Luben, Robert P. Igo Jr, Robert Wojciechowski, Ronald Klein, S. Mohsen Hosseini, Sarayut Janmahasatian, Seang-Mei Saw, Seyhan Yazar, Shea Ping Yip, Sheng Feng, Songhomitra Panda-Jonas, Stuart MacGregor, Sudha K. Iyengar, Jonathan H. Lass, Taina Rantanen, Terho Lehtimäki, Terri L. Young, Thomas Meitinger, Tien Yin Wong, Tin Aung, Toomas Haller, Veronique Vitart, Vinay 
Nangia, Virginie J.M. Verhoeven, Vishal Jhanji, Wei Chen, Xiangtian Zhou, Xiaobo Guo, Xiaohui Li, Ya Xing Wang, Yi Lu, Yik-Ying Teo, Zoran Vatavuk.

\section{References}

Abrahamsson M, Sjostrand J (2003) Astigmatic axis and amblyopia in childhood. Acta Ophthalmol Scand 81(1):33-37

Anstice J (1971) Astigmatism-its components and their changes with age. Am J Optom Arch Am Acad Optom 48(12):1001-1006

Aulchenko YS, Heutink P, Mackay I, Bertoli-Avella AM, Pullen J, Vaessen N, Rademaker TAM, Sandkuijl LA, Cardon L et al (2004) Linkage disequilibrium in young genetically isolated Dutch population. Eur J Hum Genet 12(7):527-534

Baldwin WR, Mills D (1981) A longitudinal-study of corneal astigmatism and total astigmatism. Am J Optom Physiol Opt 58(3):206-211

Bena F, Bruno DL, Eriksson M, van Ravenswaaij-Arts C, Stark Z, Dijkhuizen T, Gerkes E, Gimelli S, Ganesamoorthy D et al (2013) Molecular and clinical characterization of 25 individuals with exonic deletions of NRXN1 and comprehensive review of the literature. Am J Med Genet Part B Neuropsychiatr Genet 162(4):388-403

Boyd A, Golding J, Macleod J, Lawlor DA, Fraser A, Henderson J, Molloy L, Ness A, Ring S et al (2013) Cohort profile: the 'children of the $90 \mathrm{~s}$ '-the index offspring of the avon longitudinal study of parents and children. Int J Epidemiol 42(1):111-127

Burdon KP, Macgregor S, Hewitt AW, Sharma S, Chidlow G, Mills RA, Danoy P, Casson R, Viswanathan AC et al (2011) Genomewide association study identifies susceptibility loci for open angle glaucoma at TMCO1 and CDKN2B-AS1. Nat Genet 43(6):574-578

Capasso M, Diskin SJ, Totaro F, Longo L, De Mariano M, Russo R, Cimmino F, Hakonarson H, Tonini GP et al (2013) Replication of GWAS-identified neuroblastoma risk loci strengthens the role of BARD1 and affirms the cumulative effect of genetic variations on disease susceptibility. Carcinogenesis 34(3):605-611

Carbonaro F, Andrew T, Mackey DA, Young TL, Spector TD, Hammond CJ (2009) Repeated measures of intraocular pressure result in higher heritability and greater power in genetic linkage studies. Invest Ophthalmol Vis Sci 50(11):5115-5119

Chen Y-P, Hocking PM, Wang L, Považay B, Prashar A, To CH, Erichsen JT, Feldkaemper M, Hofer B et al (2011) Selective breeding for susceptibility to myopia reveals a gene-environment interaction. Invest Ophthalmol Vis Sci 52:4003-4011

Chen G, Ramos E, Adeyemo A, Shriner D, Zhou J, Doumatey AP, Huang H, Erdos MR, Gerry NP et al (2012) UGT1A1 is a major locus influencing bilirubin levels in African Americans. Eur J Hum Genet 20(4):463-468

Cheng C-Y, Schache M, Ikram MK, Young Terri L, Guggenheim Jeremy A, Vitart V, MacGregor S, Verhoeven Virginie JM, Barathi Veluchamy A et al (2013) Nine loci for ocular axial length identified through genome-wide association studies, including shared loci with refractive error. Am J Hum Genet 93(2):264-277

Clemons TE, Chew EY, Bressler SB, McBee W, Grp AR (2003) National Eye Institute Visual Function Questionnaire in the AgeRelated Eye Disease Study (AREDS)_AREDS report no. 10. Arch Ophthalmol 121(2):211-217

Cornes BK, Khor CC, Nongpiur ME, Xu L, Tay WT, Zheng YF, Lavanya R, Li Y, Wu RY et al (2012) Identification of four novel variants that influence central corneal thickness in multi-ethnic Asian populations. Hum Mol Genet 21(19):4365
Dirani M, Islam A, Shekar SN, Baird PN (2008) Dominant genetic effects on corneal astigmatism: the genes in myopia (GEM) twin study. Invest Ophthalmol Vis Sci 49(4):1339-1344

Dudbridge F, Gusnanto A (2008) Estimation of significance thresholds for genomewide association scans. Genet Epidemiol 32(3):227-234

Eeles RA, Kote-Jarai Z, Al Olama AA, Giles GG, Guy M, Severi G, Muir K, Hopper JL, Henderson BE et al (2009) Identification of seven new prostate cancer susceptibility loci through a genomewide association study. Nat Genet 41(10):1116-1121

Evangelou E, Ioannidis JPA (2013) Meta-analysis methods for genome-wide association studies and beyond. Nature Rev Genet 14(6):379-389

Fan Q, Zhou X, Khor CC, Cheng CY, Goh LK, Sim X, Tay WT, Li YJ, Ong RT et al (2011) Genome-wide meta-analysis of five Asian cohorts identifies PDGFRA as a susceptibility locus for corneal astigmatism. PLoS Genet 7(12):e1002402

Farbrother JE, Welsby JW, Guggenheim JA (2004) Astigmatic axis is related to the level of spherical ametropia. Optom Vis Sci 81(1):18-26

Foong AW, Saw SM, Loo JL, Shen S, Loon SC, Rosman M, Aung T, Tan DT, Tai ES et al (2007) Rationale and methodology for a population-based study of eye diseases in Malay people: The Singapore Malay Eye Study (SiMES). Ophthalmic Epidemiol 14(1):25-35

Foran S, Wang JJ, Mitchell P (2003) Causes of visual impairment in two older population cross-sections: the Blue Mountains Eye Study. Ophthalmic Epidemiol 10(4):215-225

Fraser A, Macdonald-Wallis C, Tilling K, Boyd A, Golding J, Davey Smith G, Henderson J, Macleod J, Molloy L et al (2012) Cohort profile: the avon longitudinal study of parents and children: ALSPAC mothers cohort. Int J Epidemiol 42(1):97-110

Gibb EA, Vucic EA, Enfield KSS, Stewart GL, Lonergan KM, Kennett JY, Becker-Santos DD, MacAulay CE, Lam S et al (2011) Human cancer long non-coding RNA transcriptomes. PLoS One 6(10):e25915

Grjibovski AM, Magnus P, Midelfart A, Harris JR (2006) Epidemiology and heritability of astigmatism in Norwegian twins: an analysis of self-reported data. Ophthalmic Epidemiol 13(4):245-252

Guggenheim JA, Farbrother JE (2004) The association between spherical and cylindrical component powers. Optom Vis Sci 81(1):62-63

Guggenheim JA, McMahon G, Kemp JP, Akhtar S, St Pourcain B, Northstone K, Ring SM, Evans DM, Davey Smith G et al (2013) A genome-wide association study for corneal curvature identifies the platelet-derived growth factor receptor alpha gene as a quantitative trait locus for eye size in white Europeans. Mol Vis 19:243-253

Guttman M, Amit I, Garber M, French C, Lin MF, Feldser D, Huarte M, Zuk O, Carey BW et al (2009) Chromatin signature reveals over a thousand highly conserved large non-coding RNAs in mammals. Nature 458(7235):223-227

Hammond CJ, Snieder H, Gilbert CE, Spector TD (2001) Genes and environment in refractive error: The twin eye study. Invest Ophthalmol Vis Sci 42(6):1232-1236

Han S, Chen P, Fan Q, Khor CC, Sim X, Tay WT, Ong RT, Suo C, Goh LK et al (2011) Association of variants in FRAP1 and PDGFRA with corneal curvature in three Asian populations from Singapore. Hum Mol Genet 20(18):3693-3698

Harvey EM, Dobson V, Clifford-Donaldson CE, Green TK, Messerp DH, Miller JM (2010) Prevalence of astigmatism in Native American infants and children. Optom Vis Sci 87(6):400-405

Hofman A, van Duijn CM, Franco OH, Ikram MA, Janssen HLA, Klaver CCW, Kuipers EJ, Nijsten TEC, Stricker BHC et al (2011) The Rotterdam Study: 2012 objectives and design update. Eur J Epidemiol 26(8):657-686 
Howie B, Fuchsberger C, Stephens M, Marchini J, Abecasis GR (2012) Fast and accurate genotype imputation in genome-wide association studies through pre-phasing. Nat Genet 44(8):955-959

Huynh SC, Kifley A, Rose KA, Morgan IG, Mitchell P (2007) Astigmatism in 12-year-old Australian children: comparisons with a 6-year-old population. Invest Ophthalmol Vis Sci 48(1):73-82

Hysi PG, Young TL, Mackey DA, Andrew T, Fernandez-Medarde A, Solouki AM, Hewitt AW, Macgregor S, Vingerling JR et al (2010) A genome-wide association study for myopia and refractive error identifies a susceptibility locus at $15 \mathrm{q} 25$. Nat Genet 42(10):902-905

Kassoff A, Kieval S, Mehu M, Buehler J, Eglow M, Kaufman F, Margherio RR, Cox MS, Garretson B et al (1999) The Age-Related Eye Disease Study (AREDS): design implications AREDS report no. 1. Control Clin Trials 20(6):573-600

Kassoff A, Kassoff J, Buehler J, Eglow M, Kaufman F, Mehu M, Kieval S, Mairs M, Graig B et al (2001) A randomized, placebo-controlled, clinical trial of high-dose supplementation with vitamins $\mathrm{C}$ and $\mathrm{E}$ and beta carotene for age-related cataract and vision lossAREDS Report No. 9. Arch Ophthalmol 119(10):1439-1452

Kee CS (2013) Astigmatism and its role in emmetropization. Exp Eye Res 114:89-95

Kee CS, Deng L (2008) Astigmatism associated with experimentally induced myopia or hyperopia in chickens. Invest Ophthalmol Vis Sci 49(3):858-867

Kee CS, Hung LF, Qiao-Grider Y, Ramamirtham R, Smith EL 3rd (2005) Astigmatism in monkeys with experimentally induced myopia or hyperopia. Optom Vis Sci 82(4):248-260

Keller PR, Collins MJ, Carney LG, Davis BA, Van Saarloos PP (1996) The relation between corneal and total astigmatism. Optom Vis Sci 73(2):86-91

Kelly JE, Mihashi T, Howland HC (2004) Compensation of corneal horizontal/vertical astigmatism, lateral coma, and spherical aberration by internal optics of the eye. J Vis 4(4):262-271

Khor CC, Ramdas WD, Vithana EN, Cornes BK, Sim XL, Tay WT, Saw SM, Zheng YF, Lavanya R et al (2011) Genome-wide association studies in Asians confirm the involvement of ATOH7 and TGFBR3, and further identify CARD10 as a novel locus influencing optic disc area. Hum Mol Genet 20(9):1864-1872

Kiefer AK, Tung JY, Do CB, Hinds DA, Mountain JL, Francke U, Eriksson N (2013) Genome-wide analysis points to roles for extracellular matrix remodeling, the visual cycle, and neuronal development in myopia. PLoS Genet 9(2):e1003299

Kirov G, Gumus D, Chen W, Norton N, Georgieva L, Sari M, O'Donovan MC, Erdogan F, Owen MJ et al (2008) Comparative genome hybridization suggests a role for NRXN1 and APBA2 in schizophrenia. Hum Mol Genet 17(3):458-465

Kirov G, Rujescu D, Ingason A, Collier DA, O'Donovan MC, Owen MJ (2009) Neurexin 1 (NRXN1) deletions in schizophrenia. Schizophr Bull 35(5):851-854

Klein R, Lee KE, Gangnon RE, Klein BEK (2010) The 25-year incidence of visual impairment in type 1 diabetes mellitus the wisconsin epidemiologic study of diabetic retinopathy. Ophthalmology 117(1):63-70

Lango Allen H, Estrada K, Lettre G, Berndt SI, Weedon MN, Rivadeneira F, Willer CJ, Jackson AU, Vedantam S et al (2010) Hundreds of variants clustered in genomic loci and biological pathways affect human height. Nature 467(7317):832-838

Li Y, Willer CJ, Ding J, Scheet P, Abecasis GR (2010) MaCH: Using sequence and genotype data to estimate haplotypes and unobserved genotypes. Genet Epidemiol 34(8):816-834

Li YJ, Goh L, Khor CC, Fan Q, Yu M, Han S, Sim X, Ong RT, Wong TY et al (2011) Genome-wide association studies reveal genetic variants in CTNND2 for high myopia in Singapore Chinese. Ophthalmology 118:368-375
Lopes MC, Hysi PG, Verhoeven VJM, Macgregor S, Hewitt AW, Montgomery G, Cumberland P, Vingerling JR, Young T et al (2013) Identification of a candidate gene for astigmatism. Invest Ophthalmol Vis Sci 54:1260-1267

Lyle WM (1971) Changes in corneal astigmatism with age. Am J Optom 48:467-478

Mackey DA, MacKinnon JR, Brown SA, Kearns LS, Ruddle JB, Sanfilippo PG, Sun C, Hammond CJ, Young TL et al (2009) Twins Eye Study in Tasmania (TEST): Rationale and methodology to recruit and examine twins. Twin Res Hum Genet 12(5):441-454

Mackey DA, Hewitt AW, Ruddle JB, Vote B, Buttery RG, Toomes C, Metlapally R, Li YJ, Tran-Viet KN et al (2011) Pediatric cataract, myopic astigmatism, familial exudative vitreoretinopathy and primary open-angle glaucoma co-segregating in a family. Mol Vis 17:2118-2128

Mandel Y, Stone R, Zadok D (2010) Parameters associated with the different astigmatism axis orientations. Invest Ophthalmol Vis Sci 51:723-730

Mash AJ, Hegmann JP, Spivey BE (1975) Genetic analysis of indices of corneal power and corneal astigmatism in human populations with varying incidences of strabismus. Invest Ophthalmol Vis Sci $14: 826-832$

McKean-Cowdin R, Varma R, Cotter SA, Tarczy-Hornoch K, Borchert MS, Lin JH, Wen G, Azen SP, Torres M et al (2011) Risk factors for astigmatism in preschool children the multi-ethnic pediatric eye disease and baltimore pediatric eye disease studies. Ophthalmology 118(10):1974-1981

Mishra A, Yazar S, Hewitt AW, Mountain JA, Ang W, Pennell CE, Martin NG, Montgomery GW, Hammond CJ et al (2012) Genetic variants near PDGFRA are associated with corneal curvature in Australians. Invest Ophthalmol Vis Sci 53(11):7131-7136

Mitchell P, Smith W, Attebo K, Wang JJ (1995) Prevalence of agerelated maculopathy in Australia-The Blue Mountains Eye Study. Ophthalmology 102(10):1450-1460

Mohindra I, Nagaraj S (1977) Astigmatism in Zuni and Navajo Indians. Am J Optom Physiol Opt 54(2):121-124

Nelis M, Esko T, Magi R, Zimprich F, Zimprich A, Toncheva D, Karachanak S, Piskackova T, Balascak I et al (2009) Genetic structure of Europeans: A view from the North-East. PLoS One 4(5):e5472

Oexle K, Ried JS, Hicks AA, Tanaka T, Hayward C, Bruegel M, Gogele M, Lichtner P, Muller-Myhsok B et al (2011) Novel association to the proprotein convertase PCSK7 gene locus revealed by analysing soluble transferrin receptor (sTfR) levels. Hum Mol Genet 20(5):1042-1047

Pardo LM, MacKay I, Oostra B, van Duijn CM, Aulchenko YS (2005) The effect of genetic drift in a young genetically isolated population. Ann Hum Genet 69:288-295

Parssinen O, Jauhonen HM, Kauppinen M, Kaprio J, Koskenvuo M, Rantanen T (2010) Heritability of spherical equivalent A population-based twin study among 63- to 76-year-old female twins. Ophthalmology 117(10):1908-1911

Parssinen O, Kauppinen M, Kaprio J, Koskenvuo M, Rantanen T (2012) Heritability of corneal refraction and corneal astigmatism: a population-based twin study among 66- to 79-year-old female twins. Acta Ophthalmol 91(2):140-144

Parssinen O, Kauppinen M, Kaprio J, Koskenvuo M, Rantanen T (2013) Heritability of refractive astigmatism: a population-based twin study among 63- to 75-year-old female twins. Invest Ophthalmol Vis Sci 54(9):6063-6067

Paterson AD, Waggott D, Boright AP, Hosseini SM, Shen EQ, Sylvestre MP, Wong I, Bharaj B, Cleary PA et al (2010) A genome-wide association study identifies a novel major locus for glycemic control in type 1 diabetes, as measured by both A1C and glucose. Diabetes 59(2):539-549 
Preuschhof C, Heekeren HR, Li S-C, Sander T, Lindenberger U, Bäckman L (2010) KIBRA and CLSTN2 polymorphisms exert interactive effects on human episodic memory. Neuropsychologia 48(2):402-408

Rahi JS, Cumberland PM, Peckham CS (2011) Myopia over the lifecourse: Prevalence and early life influences in the 1958 British Birth Cohort. Ophthalmology 118(5):797-804

Raitakari OT, Juonala M, Ronnemaa T, Keltikangas-Jarvinen L, Rasanen L, Pietikainen M, Hutri-Kahonen N, Taittonen L, Jokinen E et al (2008) Cohort profile: the cardiovascular risk in young finns study. Int J Epidemiol 37(6):1220-1226

Rakhshani MH, Mohammad K, Zeraati H, Nourijelyani K, Hashemi H, Fotouhi A (2012) Analysis of familial aggregation in total, against-the-rule, with-the-rule, and oblique astigmatism by conditional and marginal models in the tehran eye study. Middle East Afr J Ophthalmol 19(4):397-401

Read SA, Collins MJ, Carney LG (2007) A review of astigmatism and its possible genesis. Clin Exp Optom 90(1):5-19

Salta E, De Strooper B (2012) Non-coding RNAs with essential roles in neurodegenerative disorders. Lancet Neurol 11(2):189-200

Schippert R, Schaeffel F, Feldkaemper MP (2009) Microarray analysis of retinal gene expression in Egr-1 knockout mice. Mol Vis 15:2720-2739

Shamoon H, Duffy H, Fleischer N, Engel S, Saenger P, Strelzyn M, Litwak M, Wylierosett J, Farkash A et al (1993) The effect of intensive treatment of diabetes on the development and progression of long-term complications in insulin-dependent diabetesmellitus. N Eng J Med 329(14):977-986

Solouki AM, Verhoeven VJ, van Duijn CM, Verkerk AJ, Ikram MK, Hysi PG, Despriet DD, van Koolwijk LM, Ho L et al (2010) A genome-wide association study identifies a susceptibility locus for refractive errors and myopia at $15 \mathrm{q} 14$. Nat Genet 42(10):897-901

Spector TD, Williams FMK (2006) The UK Adult Twin Registry (TwinsUK). Twin Res Hum Genet 9(6):899-906

Sperduto RD, Hiller R, Podgor MJ, Freidlin V, Milton RC, Wolf PA, Myers RH, D’Agostino RB, Roseman MJ et al (1996) Familial aggregation and prevalence of myopia in the Framington offspring eye study. Arch Ophthalmol 114:326-332

Stambolian D, Wojciechowski R, Oexle K, Pirastu M, Li X, Raffel L, Cotch MF, Chew E, Klein B et al (2013) Meta-analysis of genome-wide association studies in 5 cohorts reveals common variants in RBFOX1, a regulator of tissue-specific splicing, associated with refractive error. Hum Mol Genet 22(13):2754-2764

Stepp MA, Gibson HE, Gala PH, Iglesia DD, Pajoohesh-Ganji A, PalGhosh S, Brown M, Aquino C, Schwartz AM et al (2002) Defects in keratinocyte activation during wound healing in the syndecan1-deficient mouse. J Cell Sci 115:4517-4531

Stepp MA, Daley WP, Bernstein AM, Pal-Ghosh S, Tadvalkar G, Shashurin A, Palsen S, Jurjus RA, Larsen M (2010) Syndecan-1 regulates cell migration and fibronectin fibril assembly. Exp Cell Res 316(14):2322-2339

Teikari JM, O’Donnell JJ (1989) Astigmatism in 72 twin pairs. Cornea 8(4):263-266

Verhoeven VJ, Buitendijk GH, Rivadeneira F, Uitterlinden AG, Vingerling JR, Hofman A, Klaver CC (2013a) Education influences the role of genetics in myopia. Eur J Epidemiol 28(12):973-980

Verhoeven VJM, Hysi PG, Wojciechowski R, Fan Q, Guggenheim JA, Hohn R, MacGregor S, Hewitt AW, Nag A et al (2013b) Genomewide meta-analyses of multiancestry cohorts identify multiple new susceptibility loci for refractive error and myopia. Nat Genet 45(3):314-318

Versteeg Gijs A, Rajsbaum R, Sánchez-Aparicio Maria T, Maestre Ana M, Valdiviezo J, Shi M, Inn K-S, Fernandez-Sesma A, Jung $\mathrm{J}$ et al (2013) The E3-Ligase TRIM family of proteins regulates signaling pathways triggered by innate immune pattern-recognition receptors. Immunity 38(2):384-398

Vitart V, Bencic G, Hayward C, Herman JS, Huffman JE, Campbell S, Bucan K, Zgaga L, Kolcic I et al (2010) Heritabilities of ocular biometrical traits in 2 Croatian isolates with extended pedigrees. Invest Ophthalmol Vis Sci 51(2):737-743

Vithana EN, Aung T, Khor CC, Cornes BK, Tay WT, Sim X, Lavanya R, Wu RY, Zheng YF et al (2011) Collagen-related genes influence the glaucoma risk factor, central corneal thickness. Hum Mol Genet 20(4):649-658

Wallman J (1994) Parental history and myopia: taking the long view. J Am Med Assoc 272(16):1255-1256

Wang S, Chen W, Chen X, Hu F, Archer KJ, Liu HN, Sun S, Gao $\mathrm{G}$ (2012) Double genomic control is not effective to correct for population stratification in meta-analysis for genome-wide association studies. Front Genet 3:300

Weale RA (2003) Epidemiology of refractive errors and presbyopia. Surv Ophthalmol 48(5):515-543

Wichmann HE, Gieger C, Illig T, Grp MKS (2005) KORA-genResource for population genetics, controls and a broad spectrum of disease phenotypes. Gesundheitswesen 67:S26-S30

Willer CJ, Li Y, Abecasis GR (2010) METAL: fast and efficient meta-analysis of genomewide association scans. Bioinformatics 26(17):2190-2191

Woodhouse JM, Adler PM, Duignan A (2003) Ocular and visual defects amongst people with intellectual disabilities participating in Special Olympics. Ophthalmic Physiol Opt 23(3):221-232 